\title{
Study on an Insulating Safeguard Based on DC Breakdown Voltages of Two Insulating Materials
}

\author{
Norimitsu Ichikawa1* and Kazuhiko Taniguchi ${ }^{2}$
}

${ }^{1}$ Department of Electrical Engineering, Kogakuin University 2665-1, Nakano, Hachioji, Tokyo 192-0015, Japan

${ }^{2}$ Department of Electrical Engineering, Kinden Corporation, Kyoto Institute, 3-1-1, Saganakadai, Kizugawa, Kyoto, 619-0223, Japan

\begin{abstract}
Fatal accidents can occur when workers do not use insulating safeguards to preventelectric shocks. Many of these accidents are caused by contact with low and highvoltage objects. These accidents may not be entirely eliminated through the use ofinsulating safeguards. Workers often fail to use safeguards because they are difficult touse in tight work situation. Therefore, a more suitable insulating safeguard is requiredto prevent these accidents. We study the design of an insulating safeguard based on theDC breakdown voltages of two insulating materials (a PMMA plate and an insulatingsheet of EVA). The breakdown voltage of the insulating sheet exhibits the polarityeffect though the characteristics of the PMMA plate are slightly dependent of thepolarity of the applied voltage. The results of this study will be useful in the basic designof insulating safeguards.
\end{abstract}

Keywords: Insulating safeguard; DC breakdown voltage; Electric shock; Insulating material

\section{Introduction}

Electric shocks are caused when electric current flows through a human body due to the contact with charged objects. Many workers worldwide are killed by electric shock. In Japan and the U.S. more than 200 fatal electric shock accidents occur annually (Figure 1). Overall, the trend of fatalities has decreased over time but has not become zero, because thorough safety training and available insulating safeguards etc. are not consistently used. Workers do not often use any insulating safeguard in the construction industry, which has the highest number of fatalities due to electric shock. Workers are often unaware of the hazard, and the available insulating safeguards are heavy and therefore difficult to use.

A more easily used insulating safeguard be developed to decrease electrical fatalities. However, to the author's knowledge, studies on the development of insulating safeguards are rarely carried out because the presently available safeguards are sufficient for all workers. However, the available safeguards are not preventing all fatalities, and we must develop safeguards that continue to decrease the fatality rate. A basis for designing insulating safeguards must be established, though

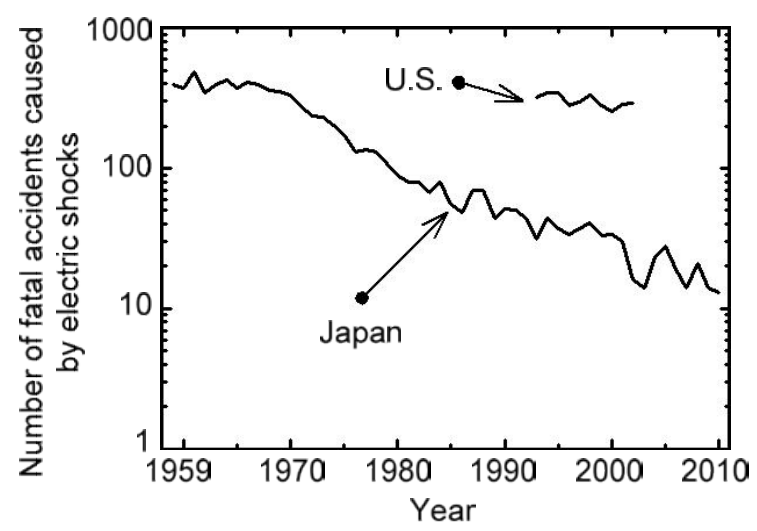

Figure 1: Fatal electric shocks in Japan and the U.S. over time [1,2]. many researchers have studied the breakdown voltages of insulating materials.

This paper describes a basis for designing an insulating safeguard based on the measured DC breakdown voltages of two insulating materials. A purpose of a study is not the comparison of breakdown voltages for same sizes of two insulating materials. The purpose is to obtain empirical formulae of breakdown voltages of two insulating materials. The study is carried out for preventing the electric shock by the contact with a DC high voltage object. The insulating materials (Figure 1) utilized here are a PMMA plate and an EVA sheet. We need a soft insulating material for designing an insulating safeguard. Hence, the EVA sheet is used. These materials are used to develop an easily utilized insulating safeguard. In experimental study, we measure the breakdown voltage on a single layer for considering the basis of design of an insulating safeguard. We can define empirical formulae that describe the breakdown characteristics of the materials (Figures 2-4). We can use these formulae todesign insulating safeguards with of predetermined breakdownvoltages to prevent electric shocks. The measured relationbetween the breakdown voltage and the creeping distance canbe applied to consider the part superimposed on two differentinsulating materials consisted of the insulating safeguard. Theincrease of the use of more easily used insulating safeguardcan prevent the case that the workers do not use the safeguard.

\section{Experimental Setup}

Figure 2 presents an arrangement of the experimental setup. The setup consists of a DC high voltage source, a source controller, and a needle plane electrode arrangement. The DC high voltage source is the

*Corresponding author: Norimitsu Ichikawa, Department of Electrical Engineering, Kogakuin University 2665-1, Nakano, Hachioji, Tokyo 192-0015, Japan, Tel: +81-42-628-4692; E-mail: ichikawa@cc.kogakuin.ac.jp

Received September 05, 2013; Accepted November 04, 2013; Published November 08, 2013

Citation: Ichikawa N, Taniguchi K (2014) Study on an Insulating Safeguard Based on DC Breakdown Voltages of Two Insulating Materials. Automat Control Physiol State Func 2: 105. doi: 10.4172/2090-5092.1000105

Copyright: (c) 2014 Ichikawa N, et al. This is an open-access article distributed under the terms of the Creative Commons Attribution License, which permits unrestricted use, distribution, and reproduction in any medium, provided the original author and source are credited. 


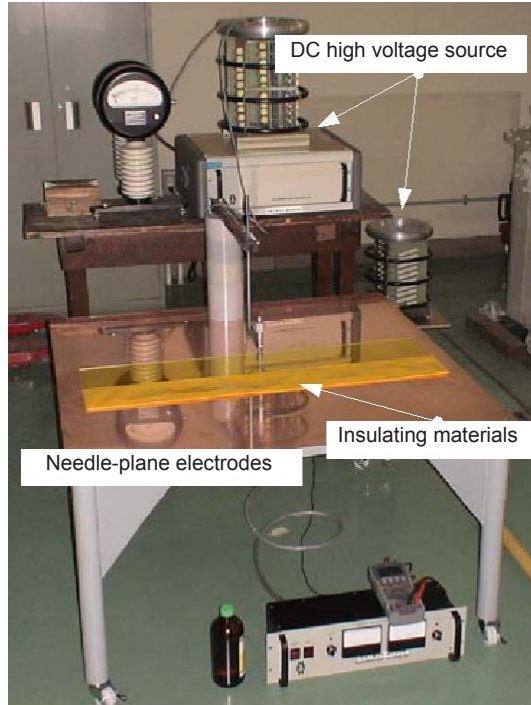

Figure 2: Arrangement of experimental setup.

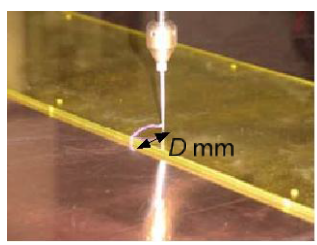

(a) PMMA plate

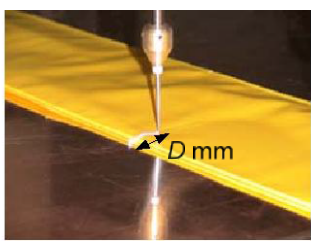

(b) Insulating sheet (EVA)
Figure 3: Arrangement of needle electrode.

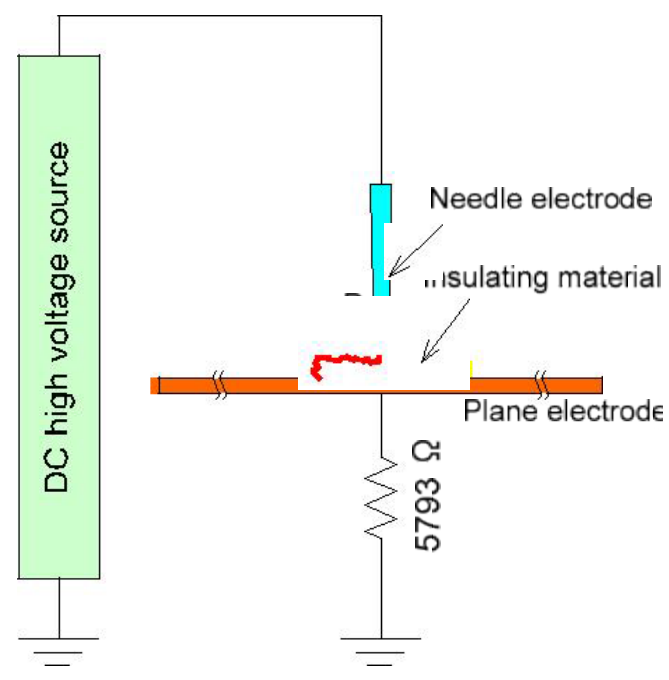

Figure 4: Diagram of experimental setup.

PS/PG100R5-10 by Glassman Co., which produces a voltage from 0 to $\pm 100 \mathrm{kV}$. The DC HV source is connected to the needle plane electrode by a high voltage cable.

A 21AAA256 (Mitsutoyo Co.) needle forms the needle part of the needle-plane electrode. The tip of the needle is a semi-sphere of $0.4 \mathrm{~mm}$ in diameter. The gap length between the needle electrode and the plane electrode can be adjusted using a micrometer head.

A plane electrode, which is a copper plate, of $1 \mathrm{~m}$ in length, $1 \mathrm{~m}$ in width, and $2 \mathrm{~mm}$ in thickness is used. The plane electrode is placed on a table of vinyl chloride. The plane electrode connects to an earth terminal through a cable with less than $10 \Omega$ of resistance in series with a protective resistor of $5793 \Omega$. The voltage between the plane electrode and the needle electrode is measured using a monitor on the DC high voltage source and an electrostatic voltmeter.

Figure 3 presents the arrangement of the needle electrode. In the experiments, two insulating materials of PMMA plate, whose dimensions are $0.15 \mathrm{~m}$ in width, $0.9 \mathrm{~m}$ in length, and $3 \mathrm{~mm}$ in thickness, and an insulating sheet of EVA (Ethylence Vinyl Acetate Copolymer), whose dimensions are $0.12 \mathrm{~m}$ in width, $0.9 \mathrm{~m}$ in length, and $1.6 \mathrm{~mm}$ in thickness, are placed on the surface of the plane electrode. The PMMA plate becomes breakable when the thickness of the plate decreases. Hence, the PMMA plate of this thickness is used. The measured relative permittivities of the PMMA plate and the EVA sheet are 2.6 and 2.3, respectively. These permittivities are measured in the frequency of 1 $\mathrm{kHz}$. The needle electrode contacts the surface of these materials as shown in Figure 4. The needle tip of the electrode is placed on the marked position of the distance $D$.

Figure 4 presents a diagram of the setup. The distance $D$ between the edge of the materials and the tip of the needle electrode is variable.

\section{Experimental Procedure}

The experiments [3] are carried out as follows (Figures 5-7).

(1) Either a PMMA plate or an EVA sheet is inserted between the needle electrode and the plane electrode.

(2) The applied voltage is increased gradually. The speed [4] of applied voltage increase is approximately $1 \mathrm{kV} / \mathrm{s}$.

(3) The breakdown voltage is measured when a spark discharge occurs.

(4) This measurement is carried out 5 times at a given distance $D$. The 5 measurements at given distance $D$ are carried out for obtaining an averaged value and a standard deviation.

The atmospheric conditions in the room are 20-21 degrees and 37$42 \% \mathrm{RH}$. The needle electrode, the plane electrode, the PMMA plate and the EVA sheet are cleaned using $\mathrm{C} 2 \mathrm{H} 5 \mathrm{OH}$ before the experiments. The cleaning is carried out for removing the dust and moisture. The needle electrode is replaced when a tip of the electrode is melted by the occurrence of few discharges.

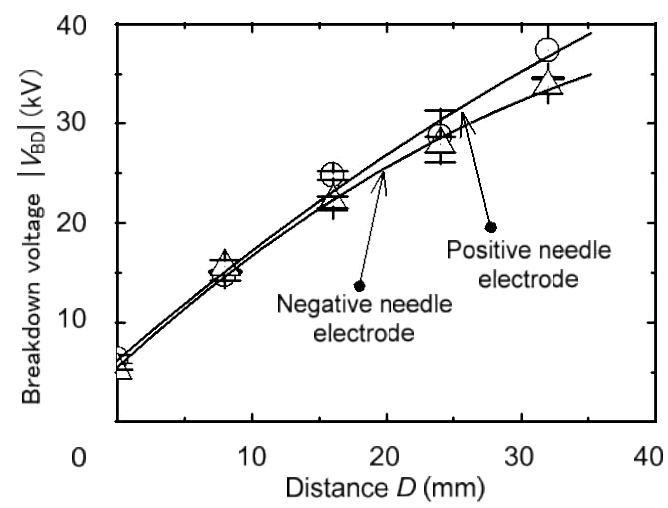

Figure 5: Characteristic of breakdown voltage for a PMMA plate. The symbol of $\circ$ represents the averaged values for the positive needle electrode, $\Delta$ represents the averaged values for the negative needle electrode. 
Citation: Ichikawa N, Taniguchi K (2014) Study on an Insulating Safeguard Based on DC Breakdown Voltages of Two Insulating Materials. Automat Control Physiol State Func 2: 105. doi: 10.4172/2090-5092.1000105

Page 3 of 4

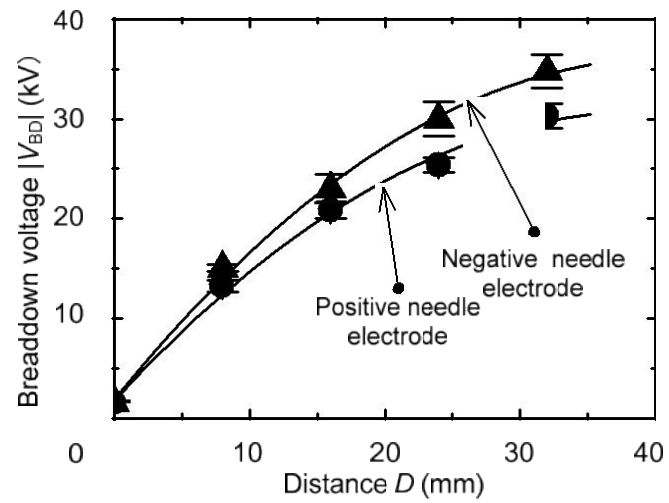

Figure 6: Charactersitics of breakdown voltage for an EVA sheet. The symbol of - represents the averaged values for the positive needle electrode, $\Delta$ represents the averaged values for the negative needle electrode.

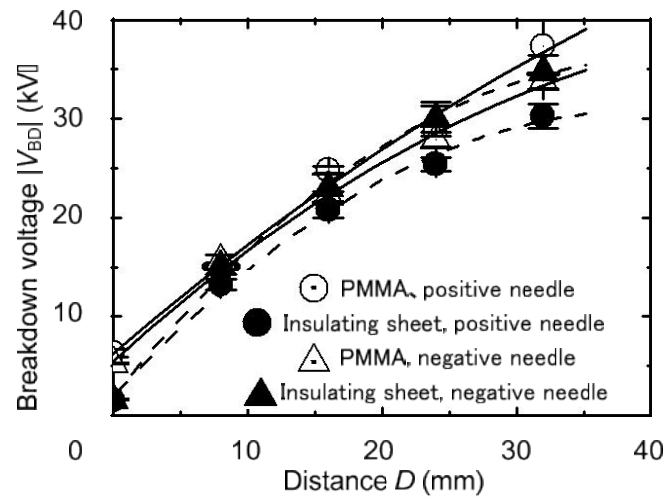

Figure 7: Comparison of breakdown voltage between a PMMA plate and an insulating sheet of EVA.

\section{Experimental Results}

A spark discharge, which is a creeping discharge, occurs between the needle electrode and the plane electrode (Figure 3) as the applied voltages increase gradually. The measured breakdown voltages for the two insulating materials are presented in Figures 5 and 6. In these figures, we compare the measured values. The error bars show the standard deviation.

Figure 5 presents the breakdown voltage vs. the distance $D$ for a PMMA plate. In the figure, the vertical axis indicates the absolute value $|V B D|$ of the applied voltage when the spark discharge occurs. The horizontal axis indicates the distance $D$ between the needle electrode and the edge of the PMMA plate. The results are measured using both a positive and a negative needle electrode. The averaged values and standard deviations are obtained from 5 repeated measurements. The minimum value of $|V B D|$ is approximately $5 \mathrm{kV}$. The value of $|V B D|$ increases as the distance $D$ increases. The breakdown voltages for a positive needle electrode are almost equal to those for a negative electrode until the distance $D$ is less than $24 \mathrm{~mm}$. The relationship between the measured breakdown voltages is summarized in Table 1.

We define the empirical formulae of Eq. (1) and (2), which can be used in the range of $0 \mathrm{~mm}$ to $32 \mathrm{~mm}$ based on the measured breakdown voltages. These empirical formulae are derived with a fitting function of the software of Origin 8.

(A) PMMA plate

(a) For a positive needle electrode:

$D \cong 0.03 \times\left(2875-2.23 \sqrt{1.85 \times 10^{6}-31600\left|V_{B D}\right|}\right)$

(b) For a negative needle electrode:

$D \cong 1.56 \times 10^{-14} \times\left(3.58 \times 10^{15}-7.66 \times 10^{6} \sqrt{2.54 \times 10^{17}-6.40 \times 10^{15}\left|V_{B D}\right|}\right)$

Where, $|V B D|$ denotes the value of the breakdown voltage $(\mathrm{kV})$, $\mathrm{D}$ denotes the distance between the tip of the needle electrode and the edge of the insulating material $(\mathrm{mm})$. These empirical formulae can be used in the range of 0 to $32 \mathrm{~mm}$ for the distance D because of the polynomial.

Figure 6 presents the measured breakdown voltages vs. the distance $D$ for an EVA sheet. The symbols in the figure are the same as those used in Figure 5. The minimum breakdown voltage $|V B D|$ is approximately $1.5 \mathrm{kV}$ at the distance $D$ of $0 \mathrm{~mm}$. The values of $|V B D|$ increase as the distance $D$ increases. The results for a positive needle electrode are almost equal to those for a negative needle electrode until the distance $D$ increases beyond $8 \mathrm{~mm}$. The breakdown voltages measured using the negative needle electrode are approximately $10 \%$ larger than those measured using the positive needle electrode at distances $D$ above 16 $\mathrm{mm}$. The relationship between the measured breakdown voltages is summarized in Table 2.

We can define the empirical formulae of Eq. (3) and (4).

(B) EVA sheet

(a) For a positive needle electrode:

$$
D \cong 0.05\left(735-1.73 \sqrt{191979-6200\left|V_{B D}\right|}\right)
$$

(b) For a negative needle electrode:

$$
D \cong 0.05\left(835-\sqrt{737601-20600\left|V_{B D}\right|}\right)
$$

Figure 7 shows the comparison of breakdown voltages between a PMMA plate and an EVA sheet. The breakdown voltage $|V B D|$ for the PMMA plate is approximately $4 \mathrm{kV}$ larger than that for the insulating sheet at the distance $D$ of $0 \mathrm{~mm}$. This difference in the thickness is related to the difference in the measured breakdown voltage.

\begin{tabular}{|c|c|c|}
\hline \multirow{2}{*}{ Distance D (mm) } & \multicolumn{2}{|c|}{ Breakdown voltage (kV) } \\
\cline { 2 - 3 } & Positive needle electrode & Negative needle electrode \\
\hline 0 & 6.3 & 5.2 \\
\hline 8 & 14.7 & 15.6 \\
\hline 16 & 24.8 & 22.1 \\
\hline 24 & 28.7 & 27.9 \\
\hline 32 & 37.3 & 33.7 \\
\hline
\end{tabular}

Table 1: Comparison of the breakdown voltages for a PMMA plate.

\begin{tabular}{|c|c|c|}
\hline \multirow{2}{*}{ Distance D (mm) } & \multicolumn{2}{|c|}{ Breakdown voltage (kV) } \\
\cline { 2 - 3 } & Positive needle electrode & Negative needle electrode \\
\hline 0 & 1.5 & 1.5 \\
\hline 8 & 13.2 & 15.0 \\
\hline 16 & 20.8 & 23.0 \\
\hline 24 & 25.4 & 30.0 \\
\hline 32 & 30.3 & 34.7 \\
\hline
\end{tabular}

Table 2: Comparison of the breakdown voltages for an EVA sheet. 
Citation: Ichikawa N, Taniguchi K (2014) Study on an Insulating Safeguard Based on DC Breakdown Voltages of Two Insulating Materials. Automat Control Physiol State Func 2: 105. doi: 10.4172/2090-5092.1000105

Page 4 of 4

\begin{tabular}{|c|c|c|c|c|}
\hline \multirow{3}{*}{ Voltage (kV) } & \multicolumn{4}{|c|}{ Distance $D(\mathrm{~mm})$} \\
\hline & \multicolumn{2}{|c|}{ PMMA plate } & \multicolumn{2}{|c|}{ EVA sheet } \\
\hline & Positive needle electrode & Negative needle electrode & Positive needle electrode & Negative needle electrode \\
\hline 10 & 3.4 & 3.8 & 5.6 & 5.3 \\
\hline 20 & 12.4 & 13.4 & 14.2 & 13.2 \\
\hline 30 & 22.7 & 26.1 & 30.1 & 24.5 \\
\hline
\end{tabular}

Table 3: The distance $D$ obtained from equations (1)-(4) for given voltages, e.g. $10 \mathrm{kV}, 20 \mathrm{kV}$, and $30 \mathrm{kV}$.

\section{Discussions}

The breakdown voltage of a negative needle electrode is approximately $10 \%$ larger than that of the positive needle electrode for the EVA sheet. The reason (polarity effect [5]) is that the progress of streamer corona becomes large by the existence of positive charges on the sheet.

We can determine a basic relationship between the breakdown voltages and the distance $D$. In Table 3 , we consider the following set of given voltages: $10 \mathrm{kV}, 20 \mathrm{kV}$, and $30 \mathrm{kV}$. When we use the equations (1)-(4), the distance $D$ in Table 3 is required.

Table 3 shows the distance $D$ used in the equations of (1)-(4) for applied voltages of $10 \mathrm{kV}, 20 \mathrm{kV}$, and $30 \mathrm{kV}$. The distance $D$ between the needle electrode and the edge of the insulating materials must be more than $6 \mathrm{~mm}$ for $10 \mathrm{kV}, 15 \mathrm{~mm}$ for $20 \mathrm{kV}$, and $31 \mathrm{~mm}$ for $30 \mathrm{kV}$. This distance $D$ can be obtained using empirical formulae.

\section{Conclusions}

In general, an insulating safeguard is made by one insulating material. Hence, the study on the safeguard by the use of two insulating materials has been seldom carried out as far as the authors know. This experimental study is carried out of the measurement of the DC breakdown voltages of a PMMA plate and an EVA sheet. The results demonstrate that the breakdown voltage of the PMMA plate is slightly dependentof the polarity of the applied voltages, while the EVA sheet exhibits the polarity effect.

We can estimate the distance $D$ required between the needle electrode and the edge of the insulating materials for a given voltage using empirical formulae. It is possible todesign an insulating safeguard that can be easily utilizedgiven the distance $D$ (Figure 8 , which presents oneexample of a basic insulating safeguard design). Theinfluence of the

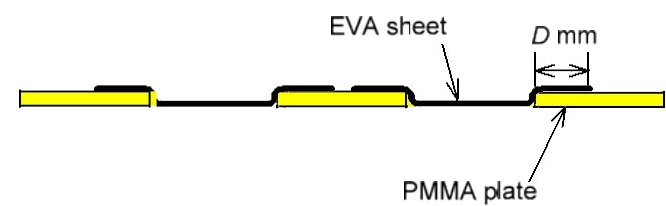

Figure 8: Example basic design of an insulating safeguard using PMMA plates and EVA sheets.

conditions of the insulating materialsurfaces on the breakdown voltage will be considered infurther studies. The breakdown voltage decreases by thesalt and the dust attached on a surface of an insulatingmaterial when the surface becomes a humid condition [6]. We will also consider the details of the insulatingsafeguards used in various industries to prevent electric shocks in further studies (Figure 8).

\section{References}

1. Cawley JC, Homce GT (2008) Trends in electrical injury in the U.S., 1992-2002. IEEE Transactions on Industry Applications. 44: 962-972.

2. Ichikawa N (2012) Japanese trend of fatal accidents caused by electric shocks and the prevention of the accidents. 2012 IEEE IAS Electrical Safety Workshop, Florida. 85-86

3. Ichikawa N (2012) Study on an insulating safeguard based upon DC breakdown voltages of insulating materials. 8th Conference of the French Society of Electrostatics, Cherboug-Octeville. 283-286.

4. Pfeiffer W (1991) High-frequency voltage stress of insulation, methods of testing. IEEE Transactions on Electrical Insulation. 26: 239-246.

5. Meek JM, Cragg JD (1953) Electrical Breakdown of Gases, Oxford, London 321-323.

6. Masamichi O (1956) Impulse flashover voltage characteristics of dielectrics in air. Journal of the Institute of Electrical Engineers of Japan. 76: 28-36. 